\title{
Evaluación e intervención pedagógica en la formación de docentes. Una acción reflexiva en el aula de clases
}

Luis Benítez Galindo

Escuela Normal de Atlacomulco "Profra. Evangelina Alcántara Díaz"

\section{Resumen}

2

1 propósito esencial de este ensayo es motivar a la reflexión y al análisis de la práctica docente, que conlleve a un proceso de autoevaluación; de tal forma que este proceso de análisis y autocrítica constante se convierta en la mejor vía de formación permanente y de perfeccionamiento de la actividad docente, especialmente cuando se hace con rigor y contando con instrumentos validos que permitan valorar en realidad la práctica; y con ello, implementar medidas correctivas que ayuden a un desarrollo pleno de la docencia. Para tal efecto, es importante que el profesor llegue a conocer su práctica, que reconozca sus aciertos y errores, e inicie una intervención a través de una lectura inteligente que le posibilite de manera estructu- rada actuar para el logro de su innovación a favor del proceso educativo. Para lograr este propósito, se asumen algunos elementos conceptuales importantes: la práctica reflexiva, la intervención pedagógica y la evaluación; concibiendo esta última como un proceso continuo del proceso enseñanza-aprendizaje. El ensayo finaliza con algunas conclusiones como son los dispositivos para el análisis y evaluación, los cuales se podrían convertir en un pretexto de discusión para las y los profesores formadores, así como para la comunidad profesional en general, por si se considera útil para cumplir con la tarea que se tiene de investigar y mejorar en forma sistemática nuestra labor docente.

Palabras clave: Intervención, evaluación, autoevaluación, docencia, estrategia. 


\section{Introducción}

El momento histórico en el que nos encontramos requiere de formar alumnos que no solo posean los conocimientos para obtener un documento que acredite que han terminado una etapa más de su formación académica, sino formar alumnos reflexivos y críticos acerca de su realidad, esto con el fin de enfrentarse a ella y salir adelante en la tarea que decidan emprender.

Independientemente de los cambios y exigencias que se producen, es importante motivar el cambio de pensamiento en los profesores, ya sea a nivel de actitud o conceptual, además de la forma de percibir y entender las relaciones complejas entre el sujeto y objeto de la educación, así como los fines de la misma, de modo que permitan transformar la escuela, la enseñanza y el aprendizaje.

Dentro de este conjunto de reformas y cambios, la más polémica y controversial se relaciona con el proceso de evaluación sobre el desempeño del trabajo docente, más aún si se trata de cambiar e innovar la práctica en el aula de clase. Generalmente sólo medimos el rendimiento de los estudiantes y hacemos juicios a prior $i^{1}$, tales como: "los estudiantes son malos", "los alumnos no estudian", "los alumnos son apáticos", o "los alumnos no tienen interés por la clase", sin antes detenernos a pensar, cuestionar, reflexionar y analizar que, en muchas ocasiones, el desempeño del profesor influye de alguna forma en ese mal rendimiento estudiantil. Por lo tanto, considero necesario motivar a los y las colegas para crear una cultura de autoevaluación y reflexión de la práctica docente, contribuyendo así a mejorar los procesos educativos y la práctica misma.

Con esto pretendo compartir una postura hermenéutica para la valoración de la práctica docente, la cual comprende los tres momentos de toda actividad educativa: previo, durante y posterior a la tarea, lo que permitirá hacer un alto en el camino para analizar y autocriticar la práctica que realizamos a través de nuestras iniciativas pedagógicas, de tal forma que a partir de los resultados del autoanálisis, el profesor identifique potencialidades y problemas en su ejercicio cotidiano con el objetivo de determinar necesidades de retroalimentación, perfeccionamiento o de actualización $y$, en ese sentido, proponer un plan estratégico individual y personal que permita mejorar la tarea y el acto educativo a corto, mediano y largo plazo.

\section{Saber reflexionar sobre la propia práctica}

En plena acción pedagógica, hay poco tiempo para meditar, por lo que casi al instante se tiene que reflexionar para dar el siguiente paso, para decidir el camino que se debe seguir; por ejemplo, interrumpir o no una conversación entre alumnos, empezar o no con un nuevo contenido temático antes de acabar la clase, aceptar o no una excusa, castigar a un alumno indisciplinado o el que llega tarde a una sesión de clase, responder o no a una pregunta insolente, incongruente o fuera de lugar, dejar salir o no a un alumno durante el desarrollo de un tema, etc.

Cada una de estas "microdecisiones" pone en marcha una actividad mental. In- 
cluso cuando nos encontramos en la rutina, la actividad aparece pre reflexionada, al límite de la conciencia. Pensamos sin ser conscientes qué pensamos; es decir, no hay deliberación interior, no hay dudas, por lo tanto, se dirá: no hay reflexión, en el sentido propio de la acción. Sin embargo, a veces surge la incógnita y sopesamos entre dos posibilidades, entre los impulsos contradictorios, entre un movimiento afectivo y la razón emocional que los impulsa.

Cuando no sabemos muy bien lo que hay que hacer, vistas las circunstancias, el tiempo que transcurre y qué falta por aprovechar, el clima de la clase, el trabajo iniciado, se puede disparar una reflexión en plena acción, cuando incluso el flujo de los acontecimientos no se interrumpe e impide una verdadera paralización de la acción. No intervenir es entonces también una forma de actuar, en el sentido en que esta actitud pesará así mismo, de otra manera, en el curso de los acontecimientos. Si no decidimos nada jamás, dejamos que la situación evolucione $y$, tal vez, que empeore.

La reflexión en la acción, "es y debe ser rápida, porque guía un proceso de decisión, sin recurso posible a opiniones externas, sin la posibilidad de pedir un tiempo muerto, como un equipo de baloncesto tiene derecho a hacerlo durante un partido"(Perrenoud, 2007:33).

Este proceso puede llevar a la decisión de no intervenir inmediatamente y darse tiempo para reflexionar con más tranquilidad. Es lo que Bordieu (1991) siguiere a los docentes, al invocar un "saber de inacción", que se puede interpretar como una forma de sabiduría incorporada al habitus ${ }^{2}$, la cual conduce a diferenciar la decisión. No toda indecisión es fatal, incluso, en algunas situaciones, justifican una respuesta anterior.

El profesor siente que actuar demasiado de prisa sería poco acertado, pues se encuentra bajo los efectos de la emoción y le faltan elementos de apreciación para decidir con conocimiento de causa sobre el problema. A veces, puede decidir abiertamente ante la presencia de sus alumnos: "Pues no lo sé. Voy a pensar en ello y responderé mañana". En otras situaciones, esta reflexión es interior.

En clase, algunos comportamientos sólo se convierten en problemas porque son repetitivos, por ejemplo: los charloteos crónicos del profesor o profesora cuando no hay preparación previa de una clase formal, la impuntualidad constante a una sesión programada, la huida continua ante las tareas, las agresiones repetidas en alumnos y compañeros o la impertinencia habitual. Entonces, la decisión no nos lleva a una situación singular, pero sí a una serie de situaciones parecidas, lo que deja tiempo para formarse una opinión o contemplar diversas estrategias posibles.

La reflexión en la acción permite decidir si tenemos que actuar inmediatamente o si podemos darnos algo de tiempo para una reflexión más tranquila sin caer en la provocación de la incertidumbre. Si revisamos el análisis que realiza Schön (1998), ahí se advierte que desarrollar una práctica reflexiva significa aprender a aprovecharse de la reflexión gracias a un ajuste de los esquemas de acción, que permita una intervención más rápida, más concreta $o$ 
más segura; un esfuerzo de la imagen de uno mismo como profesional reflexivo en proceso de evolución, y un saber integrado, que permitirá comprender y dominar otros problemas profesionales.

Por lo tanto, la práctica reflexiva también significa, desde la formación inicial, hacer que los alumnos entren en contacto con el sector más activo del oficio o la profesión, si bien estos futuros enseñantes consideran que la etiqueta de practicantes reflexivos resulta pesada de cargar o simplemente demasiado tecnocrática o restrictiva.

\section{Intervención en la práctica pedagógica}

El profesor, considerado como el mediador o acompañante estratégico entre los estudiantes para promover, orientar, descubrir y construir sus propios aprendizajes, juega un papel importante y decisivo en la promoción de los procesos de crecimiento personal del alumno y en el marco de la cultura grupal a la que pertenece. La intervención (del latín interventio) "es venir entre, interponerse: la intervención es sinónimo de meditación o de intersección, de buenos oficios, de ayuda, de apoyo, de cooperación. Se le atribuye el uso de las ideas de operación y de tratamiento" (Maher y Zins, 1987:168).

En esta misma línea, Rodríguez (1988) define el concepto de intervención como guiar, conducir e indicar (de manera procesual) para ayudar a las personas a conocerse a sí mismas y al mundo que las rodea; es auxiliar a un individuo a clarificar la esencia de la vida, a comprender que él es una unidad con significado capaz de y con derecho a usar su libertad, su dignidad personal, dentro de un clima de igualdad de oportunidades y actuando en calidad de ciudadano responsable, tanto en su actividad laboral como en su tiempo libre.

Esto es un proceso de acompañamiento uniforme durante la formación de los alumnos, con un académico preparado y formado para esta función. Por esta razón, se considera que la intervención es una labor de carácter preferentemente especializada que, en gran medida, coincide con un subconjunto de la orientación educativa.

Entonces, intervenir en la práctica implica un conocimiento extenso de la misma. El profesor con intención y espíritu de mejorar su práctica, será un riguroso investigador de sí mismo, y se pondrá en acción de manera planeada y con sustento en su propia investigación; además, desarrollará un proyecto que conlleve estrategias, actividades y acciones que transformen significativamente su quehacer diario en atención a los procesos educativos de los alumnos.

Cuando se está investigando la práctica docente y uno se enfrenta con el reconocimiento de una serie de problemas que demuestran que el quehacer del profesor tiene deficiencias, es necesario elaborar un plan de intervención para resolver la problemática de su praxis.

Al reflexionar, analizar y cuestionar la práctica, se infiere sobre cuáles serían los posibles problemas del docente. Este reconocimiento de problemas, aunado a la historia de vida, demuestra las dificultades del profesor y, por lo tanto, la construcción del aprendizaje por parte de los alumnos se ve totalmente limitada. Para la problematiza- 
ción de la práctica, es preciso poner sobre la mesa los hallazgos, analizarlos, compararlos, reflexionarlos y, así, dar cuenta de las relaciones que existen entre ellos; verificar que no son aislados y conforman una red que representan la problemática del profesorado.

Para cerrar la problematización es necesario haber reflexionado a profundidad la práctica, desde la cual emerge una pregunta, con el afán de intervenir y cambiar aquello que no está generando el propósito educativo, dando pie a una propuesta de intervención ${ }^{3}$. Sin embargo, el diseño de un plan investigativo de intervención es el proceso de planificar acciones dentro de un marco conceptual y metodológico, que instrumente el seguimiento, la recuperación, el análisis y la producción del conocimiento derivado del proceso de transformar una cultura pedagógica dentro de una situación institucional.

El plan de intervención incluye sus propias mediciones pertinentes, no al proceso de la investigación, sino al de la intervención misma, aunque estas dos dimensiones deben responder a criterios de coherencia interna en cuanto a sus planteamientos epistemológicos, teóricos y metodológicos. Para realizar el plan de intervención es necesario plantearse una pregunta que vaya encaminada a resolver el problema reconocido, de tal forma, que la intervención sea intencionada y se formulen las estrategias que resuelvan la interrogante, aunadas a actividades y acciones que complementen el proceso del profesor como interventor de su propia práctica, con capacidad y profesionalismo.
La estrategia didáctica es, por lo tanto, un sistema de planificación aplicable a un conjunto articulado de acciones para llegar a una meta. De manera que no se puede hablar del uso de estrategias cuando no hay una meta hacia donde se orienten las acciones. La estrategia ${ }^{4}$ debe fundamentarse en un método; pero a diferencia de éste, la estrategia es flexible y puede tomar forma con base en las metas a donde se quiere llegar.

En su aplicación, con la estrategia se puede hacer uso de una serie de técnicas para conseguir los objetivos perseguidos. De ahí que en la estructura de un plan se deben considerar los siguientes elementos:

Título, antecedentes y descripción del contexto educativo; Diagnóstico pedagógico del grupo (detección del área de intervención); Fundamentación y justificación (¿por qué? y ¿para qué?); Diseño del programa de intervención (objetivos, contenidos, actividades de enseñanza y/o aprendizaje, material de apoyo, duración de la intervención, estrategias de evaluación (instrumentos de seguimiento e instrumentos de evaluación); Fase operativa (procedimiento de aplicación, eventualidades y su resolución); Evaluación de los resultados de la aplicación de programa y conclusiones.

Al seguir una estructura semejante, es posible desarrollar un proyecto de intervención, ya que el docente competente sabrá construir saberes competentes para gestionar situaciones profesionales, cada vez más creativas e innovadoras.

Hablaré un poco del término competencia desde el paradigma interpretativo. 
Le Boterf (2000) nos previene contra una definición débil de las competencias (suma de conocimientos de saber hacer o saber estar o como la aplicación de conocimientos teóricos o prácticos) y nos recuerda que las competencias no son un conglomerado de conocimientos fragmentados, ni está hecha de migajas de saber hacer, sino que es un saber combinatorio y que no se trasmite. El centro de la competencia es el sujeto-aprendiz, quien la construye a partir de una secuencia de las actividades de aprendizaje que movilizan múltiples conocimientos especializados.

No obstante, si nos preguntamos $i^{\mathrm{a}}$ quiénes corresponde hoy día reorganizar la práctica educativa?, parecería imposible prescindir de la tradición pedagógica como uno de los modelos posibles de la práctica reflexiva. Seguramente, es en este ámbito, donde debemos buscar las herramientas reflexivas formalizadas, pues es allí donde hallaremos en los escritos de grandes pedagogos: Dewey, Freire, Freinet, Makarenko, Montessori, Pestalozzi, Decroly, Illich, Neil, Herbart o Rousseau, la encarnación de la postura reflexiva, de la posición de comprender, del ir y venir obstinado e incesante entre la teoría ${ }^{5}$ y la práctica, de la observación regular, de recuperar el oficio y la vocación a base de observación e hipótesis.

\section{Principios de la de evaluación y la toma de conciencia sobre el trabajo docente}

¿Quién no ha hablado de la evaluación en estos últimos tiempos? ¿Quién no ha discutido con colegas sobre el tema? La evaluación está omnipresente, devinién- dose en algo común, sin embargo, permanece poco conocida, mal comprendida y a menudo mal interpretada. Constituye un término polisémico, una especie de imán semántico, situado en un campo de múltiples interrogantes.

Si bien es una necesidad indiscutida, las reacciones que suscita van desde la adhesión al rechazo, pasando por la aprensión donde la gran interrogante estribaría ¿de qué estamos hablando cuando hacemos mención el término evaluación? En ese sentido, cobran vital importancia la evaluación de la práctica docente, el proceso de autoevaluación, la evaluación de las instituciones, los criterios para construir evaluaciones y los sistemas de calificación.

La evaluación es un elemento esencial que debe aplicarse tanto al proceso de aprendizaje de cada alumno como a la revisión de la práctica docente. El término evaluación ha sido enfocado y analizado desde diferentes puntos de vista y enfoques, uno de ellos es el citado por Gutiérrez Cerda quien plantea que:

En el caso de la investigación acción, esquema de racionalización de los procesos didácticos, donde se resalta la importancia de la reflexión de lo ocurrido, la evaluación es un recurso para mejorar los procesos pedagógicos. En estos planteamientos ésta tiene el significado y valor de servir a la toma de conciencia sobre la práctica; es decir, sirve para pensar y planificar la práctica pedagógica (2002).

Gimeno Sacristán y Pérez Gómez (1996) la plantean como un proceso continuo complejo y global que nos permite recoger sistemáticamente información relevante, tanto de los resultados y logros en el apren- 
dizaje como de la intervención docente en el proceso educativo, con el objeto de reajustar la intervención de acuerdo con los aprendizajes reales y necesidades del alumnado, y no simplemente adjudicar un número o nota; a la vez permite identificar las competencias y la práctica docente, la cual requerirá de modificaciones y adecuaciones según sea el caso.

Por lo tanto, la evaluación no es un fin en sí misma, sino apenas un instrumento, medio o herramienta para mejorar el trabajo y los resultados o puntos de referencias para emitir un juicio que sea relevante para la intervención pedagógica-educativa. La evaluación y autoevaluación docente deben necesariamente cumplir con determinados propósitos:

- Ayudar a los maestros a encontrar vías que desarrollen sus destrezas profesionales.

- Ayudar a la planificación del perfeccionamiento y desarrollo profesional individual y colectivamente.

- Identificar el potencial del maestro para el desarrollo profesional, con la intención de ayudarles a través de la educación en servicio y orientación adecuada, entre otras.

Visto así, la evaluación debe realizarse en todo momento, antes, durante y después del acto educativo; y ser acorde con el plan o proyecto curricular, los objetivos, contenidos y perfil educativo o de formación. También es importante saber que su función es identificar los logros y las debilidades del alumno, el docente y la institución educativa.
Para que cualquier profesor asuma, de buena manera, la tarea de cambiar sus pautas de comportamiento, es primordial que lo haga de manera voluntaria, aunque se le haya sugerido. Ya que el comportamiento de los profesores, como cualquier otro, es motivado. En este sentido, la evaluación es un factor de impulso para la trasformación de la práctica pedagógica.

Cuando hacemos algo, es por alguna razón y con algún propósito más o menos consciente. $\mathrm{O}$ bien, puede deberse a un comportamiento dirigido por nuestros conocimientos, de los que somos más o menos conscientes, pues nuestra forma de actuar puede estar automatizada sobre la funcionalidad y relevancia de lo que hacemos para conseguir las distintas metas que perseguimos.

Por lo tanto, es necesario valorar los momentos didácticos del hecho pedagógico: preparación, ejecución, desarrollo y evaluación, los cuales sirven para orientar la evaluación de la práctica, así como para que el profesor valore y tenga la oportunidad para pensar el desarrollo en cada espacio de su quehacer.

Profesionalizar la práctica docente implica asumir una postura reflexiva por parte de los educadores, ya que desarrollar una postura reflexiva significa tomar conciencia y fomentar la instauración de los esquemas reflexivos para intervenir sin prejuicios a la hora de colaborar y responder a las diversas necesidades de los educandos al convivir, comunicarse y relacionarse en la resolución de problemas.

En este sentido, Bourdieu (1981) reconoce que el habitus es un sistema de es- 
tructuras de pensamiento, de percepción, de evaluación y de acción de nuestras prácticas. De aquí que sea importante formarlo, como un punto básico entre los saberes y las situaciones que exige una acción.

Esto significa que la intervención y evaluación son parte integrante de nuestra vida, ya que continuamente somos sujetos de evaluación de lo que realizamos. No pasa un día sin que al finalizar la jornada no hagamos un balance de lo que hicimos y cómo lo hicimos, o qué nos falta por hacer. Así sucede en todos los aspectos de la vida. Por ello, no es posible pensar y organizar un proceso de enseñanza sin que esté presente el componente de una evaluación documentada, de ahí que la evaluación deba ser congruente con el aprendizaje.

\section{Conclusiones}

Cuando se pretende la excelencia en el desempeño educativo es indispensable continuamente evaluar nuestros propios procedimientos de enseñanza, de modo que nos permita adecuarlos a las necesidades y características del alumnado y, así, satisfacer sus expectativas como sujetos en formación. De igual modo, los alumnos deben ser sometidos sistemáticamente a evaluaciones para poder comprobar su evolución, así como la eficacia de nuestro propio desempeño profesional.

Las escuelas se componen de profesionales quienes deben buscar constantemente servir a sus alumnos y a la comunidad de la mejor manera posible; deben poseer unos criterios exigentes altos; deben comparar sus prácticas bajo el consenso y el referente teórico relacionado con las compe- tencias que los profesores deben desarrollar para profesionalizar la labor docente y; deben intentar mejorar aquellos aspectos de su trabajo que sean deficientes, o cuando el estado de la ciencia haya confirmado que existen mejores estrategias para educar de otra manera a sus alumnos.

Es importante que los maestros se adapten a los alumnos, para lo cual deben conocerlos, y discernir la manera de proceder con cada uno. Y si hubiera que reducir toda la psicología educativa a un sólo principio, quedaría este: el factor más importante que influye en el aprendizaje es lo que el alumno ya sabe. Es la razón del auge que tiene en la actualidad la evaluación inicial y la dimensión diagnóstica de la evaluación.

También hay que trabajar en colaboración con colegas, padres y la comunidad, con el fin de satisfacer de manera efectiva el abanico de necesidades educativas de todos sus alumnos y mostrarse abiertos e interesados en que otros evalúen su trabajo.

El trabajo reflexivo y la profesionalización de la práctica docente implican una ética coherente y una gran capacidad conceptual, que permiten saber dónde está el límite que conduce la intervención, la participación, la opinión, la libertad, el riesgo y la responsabilidad, pero sobre todo, la comunicación racional y humanización colectiva en las ideas con los otros como sujetos sociales.

Finalmente, una escuela efectiva es aquella cuyos profesionales realizan y utilizan, sistemáticamente, las evaluaciones para servir de manera eficiente a los alumnos y para informar al público sobre los logros y necesidades de la escuela. 
Desde este punto de vista, el desarrollo de una postura y prácticas reflexivas más extendidas, constantes e instrumentadas, son la clave en la mejora de la intervención en el aula y fuera de ella; por lo tanto, un principio y esperanza para salir, de forma progresiva, de la situación del punto muerto a la que nos conducen nuestros pensamientos de corte técnico-instrumental para hacer frente al nuevo paradigma lingüístico de las reformas escolares del siglo XXI es: formar a los estudiantes en la convicción y capacidades necesarias para contribuir a la construcción de una sociedad más justa e incluyente.

El planteamiento de Morín (2007) se inscribe precisamente en este sentido: el mundo se moverá en una dirección ética, sólo si queremos ir en esa dirección. Y serán nuestra responsabilidad y nuestro destino el que está en juego. El pensamiento complejo es una aventura, pero también un desafío.

Así, la intervención no es solo una propuesta, sino un movimiento con múltiples propuestas fundado en una acción intencional de investigación que intenta abrir líneas de reflexión para incrementar el conocimiento del problema educativo: cómo se aprende, cómo se educa y cuándo, qué sucede dentro de los espacios que cobijan el hecho educativo; y, por tanto, qué modificaciones metodológicas debe hacer el maestro en sus propias prácticas para mejorar la oferta educacional concreta para que los educandos avancen en su formación integral.

\section{Notas}

1- Conocimientos derivados de fuentes de experiencia. La experiencia es sin duda el primer producto que elabora nuestro entendimiento, con la materia bruta de las percepciones sensibles. Precisamente por eso es la primera enseñanza $y$, en el progreso, se muestra tan inagotable en nuevas enseñanzas, que le encadena vida a todas las futuras producciones de nuevos conocimientos, que puedan juntarse en ese suelo, no tendrá nunca falta. Sin embargo no es ni con mucho el único campo en donde se deja limitar nuestro entendimiento. Nos dice, es cierto, lo que existe, pero no que ello tiene necesariamente que ser así y no de otro modo. (Kant, 2005:30).

2- Podemos llamar habitus al conjunto de los esquemas de que dispone una persona en un momento de su vida. También se define como un "pequeño conjunto de esquemas que permite engendrar infinidad de prácticas adoptadas a situaciones siempre renovadas, sin constituirse jamás en principios explícitos" (Bourdieu, 1972:209).

3- Un propuesta de intervenciónse considera como una propuesta factible, creativa y detallada y su aplicación, para realizar una mejora o resolver una problemática grupal, social, institucional y empresarial, sobre cualquier aspecto que afecte a su buen desempeño, por lo tanto, el punto de arranque de toda propuesta investigadora en el campo educativo surge cuando se plantea una pregunta o un interrogante al que no podemos ofrecer una solución satisfactoria. (Bisquerra, 2003:92-93).

4- La palabra estrategia viene del griego y significa "artede ganar la guerra". Una estrategia es por consiguiente, en un sentido estricto, el arte o la forma de lograr el objetivo; en palabras de Monereo (1997), un procedimiento organizado, formalizado y orientado a la obtención de una meta claramente establecida. Su aplicación en la práctica diaria requiere del perfeccionamiento de procedimientos y de técnicas cuya elección detallada y diseño son responsabilidad del docente.

5- Desde la definición científica, una teoría, es un conjunto de conceptos (constructos), definiciones y proposiciones vinculadas entre sí, que presentan un punto de vista sistemático del fenómeno al especificar las relaciones entre variable, con el objetivo de explicar y predecir los fenómenos (Kerlinger y Lee, 2002).

\section{Bibliografía}

Bisquerra, A. R. (2003) Modelos de orientación e intervención psicopedagógica. Barcelona: CISS Praxis.

Bisquerra, A. R. (1998) Intervención y orientación psicopedagógica. Barcelona: CISS Praxis. 
Bourdieu, P. (1972) Esquissed'unethéorie de la practique. Genéve: Droz

Bourdieu, P. (1991) El sentido práctico. Madrid: Taurus.

BOURDIEU, P. (1981) La reproducción: elementos para una teoría de enseñanza. Barcelona: Laia.

DíAz BArriga, A. F. (2006) La enseñanza situada. Vínculo entre la escuela y la vida. México: Mc Graw Hill.

Elliott, J. (1990) El cambio educativo desde la investigación-acción. Madrid: Morata.

Gimeno, S. J. y PÉRez, G. A. I. (1996) Comprender y transformar la enseñanza. Madrid: Morata.

GutiérRez, C. H. (2002) La evaluación como experiencia total. Logros, objetivos, procesos, competencia y desempeño. Santa Fe de Bogotá: Nomos.

Jesús Ma, N. G. (1996) La Autoevaluación del profesor. Madrid: Española.

Kant, M. (2005) Crítica de la razón pura. México: Porrúa.

Maher, C. A. Y Zins, J. E. (1987) Intervención psicopedagógica en los centros educativos. Madrid: Narcea.
Monereo, C. (1997) Estrategias de enseñanza y aprendizaje. Formación del profesorado y aplicación en el aula. México: Biblioteca Normalista.

Morín, E. (2000) Introducción al pensamiento complejo. Barcelona: Gedisa.

Le Boterf, G. (2000) Ingeniería de las competencias. Barcelona: Gestión 2000/EPISE.

Perales, P. R. C. (2009) La significación de la práctica educativa. México: Paidós.

Rodríguez, M. L. (1988) Orientación educativa. Barcelona: Ceas.

Perrenoud, P. (2007) Desarrollar la práctica reflexiva en el oficio de enseñar. Barcelona: Graó.

Kerlinger, F. N. Y LeE, H. B. (2002) Investigación del comportamiento: Método de investigación en ciencias sociales. México: McGraw-Hill.

Schôn, D. (1998) El profesional reflexivo: cómo piensan los profesionales cuando actúan. Barcelona: Paidós.

Zabala, V. A. (2006). La práctica educativa. Cómo enseñar. México: Grao. 